\title{
Anticancer and radiosensitizing effects of the cyclin-dependent kinase inhibitors, AT7519 and SNS-032, on cervical cancer
}

\author{
MI AE KANG ${ }^{1 *}$, WONWOO KIM ${ }^{2 *}$, HYE-RAM JO ${ }^{1,3}$, YOUNG-JOO SHIN ${ }^{4}$, \\ MOON-HONG KIM ${ }^{5}$ and JAE-HOON JEONG ${ }^{1,3}$ \\ ${ }^{1}$ Division of Applied Radiation Bioscience, and ${ }^{2}$ Radiation Non-Clinic Center, Korea Institute of Radiological \\ and Medical Science, Seoul 01812; ${ }^{3}$ Radiological and Medico-Oncological Sciences, \\ Korea University of Science and Technology, Daejeon 34113; ${ }^{4}$ Department of Radiation Oncology, \\ Inje University Sanggye Paik Hospital, Seoul 01757; ${ }^{5}$ Department of Obstetrics and Gynecology, \\ Korea Institute of Radiological and Medical Sciences, Seoul 01812, Republic of Korea
}

Received January 30, 2018; Accepted May 17, 2018

DOI: $10.3892 /$ ijo.2018.4424

\begin{abstract}
Cyclin-dependent kinases (CDK) are considered to be potential targets of anticancer drugs that can interrupt the uncontrolled division of cancer cells. In this study, we selected two selective CDK inhibitors, AT7519 and SNS-032, from current clinical trials and examined their anticancer and radiosensitizing effects in a cervical cancer model. SNS-032 was found to be more potent than AT7519, with a lower half maximal inhibitory concentration $\left(\mathrm{IC}_{50}\right)$ value. Both AT7519 and SNS-032 induced the apoptosis, premature senescence and cytostasis of cervical cancer cells, which led to the attenuation of tumor growth in vivo. Moreover, using these CDK inhibitors together with radiation synergistically inhibited tumor growth in a human xenograft tumor model. The concomitant activation of the p53 tumor suppressor and the suppression of cell cycle checkpoint responses mediated by $\mathrm{Chk} 1$ led to the cytostasis of cervical cancer cells. Finally, AT7519 and SNS-032 inhibited cancer cell migration, invasion and angiogenesis in vitro, and suppressed lung metastases in a spontaneous metastasis model. On the whole, the findings of this study indicate that
\end{abstract}

Correspondence to: Dr Jae-Hoon Jeong, Division of Applied Radiation Bioscience, Korea Institute of Radiological and Medical Sciences, 75 Nowon-ro, Nowon-Gu, Seoul 01812, Republic of Korea E-mail: jeongj@kirams.re.kr

Dr Moon-Hong Kim, Department of Obstetrics and Gynecology, Korea Cancer Center Hospital, 75 Nowon-ro, Nowon-gu, Seoul 01812, Republic of Korea

E-mail: garymh@kcch.re.kr

${ }^{*}$ Contributed equally

Abbreviations: CDK, cyclin-dependent kinase; SA, senescenceassociated; IR, ionizing radiation

Key words: cyclin-dependent kinase, AT7519, SNS-032, apoptosis, senescence, cytostasis, radiosensitization, metastasis the utilization of AT7519 and SNS-032 as part of an adjuvant treatment may help control cervical cancer progression.

\section{Introduction}

Cyclin-dependent kinases (CDKs) are present in all known eukaryotes, and their regulatory functions during the cell cycle are evolutionarily conserved. Cyclin-CDK complexes phosphorylate specific substrates, according to the requirements of a particular cell cycle phase. CDKs are regulated by cyclin binding, phosphorylation and the binding of CDK inhibitors (1). In addition to cell cycle regulation, CDKs are involved in transcription, mRNA processing and cellular differentiation (2-4).

In a number of human cancer types, CDKs are overactive and CDK-inhibiting proteins are non-functional $(5,6)$. Therefore, CDKs are considered potential targets for anticancer therapies, by interfering with CDK functions to selectively interrupt cell cycle regulation in cancer cells (7). Flavopiridol (alvocidib) was the first CDK inhibitor to be tested in clinical trials following its identification in a screen for anticancer agents in 1992. It competes for the ATP-binding site of CDKs (8). Although CDK inhibitors seem therapeutically promising, their side-effects must be limited so that only cancer cells are affected. AT7519, a pyrazole 3-carboxyamide compound, was developed by Astex and acts as an inhibitor of CDK1, CDK2, CDK4, CDK6 and CDK9. Santo et al showed demonstrated AT7519 exerts potent cytotoxic effects and induces the apoptosis of multiple myeloma cells; AT7519 was also found to be associated with the inhibition of in vivo tumor growth and prolonged survival (9). AT7519 has also been clinically evaluated in a phase I study of patients with advanced refractory solid tumors or non-Hodgkin's lymphoma (10), and in a phase II clinical trial (NCT01183949). SNS-032 was developed by Bristol-Myers Squibb; this compound exhibits potent and selective inhibitory activity against CDK2, CDK7, and CDK9. Chen et al demonstrated that SNS-032 effectively killed chronic lymphocytic leukemia cells in vitro regardless of prognostic indicators and treatment history (11). Two phase I clinical studies of SNS-032 have been reported $(12,13)$; however, no further developments have been reported. 
CDKs are not only required for proper cell cycle progression, but are also involved in DNA damage repair, particularly in the repair pathway choice between homologous recombination (HR) and non-homologous end joining (NHEJ) (14). The phosphorylation of breast cancer 2 tumor suppressor (BRCA2) by CDK inhibits its interaction with RAD51 and regulates the HR pathway for double-strand DNA repair (15). In addition, eukaryotic cells have been shown to respond to DNA lesions via the activation of a complex signal transduction pathway, known as the DNA damage checkpoint, which delays cell cycle progression, while stimulating DNA repair (16). It has also been reported that CDK1 inhibition abrogates S-phase cell cycle arrest and the inefficient phosphorylation of ataxia telangiectasia mutated (ATM)/ataxia telangiectasia and Rad3 related (ATR) substrates, leading to the inhibition of BRCA1 recruitment to the DNA damage foci (17). Although the activation of DNA damage signals inhibits CDK complexes and prevents cell cycle progression, CDK activity is also required for checkpoint recovery through the activation of the Forkhead transcription factor FoxM1 $(18,19)$. These studies suggest that the abrogation of HR repair and checkpoint control by targeting CDKs can cause cellular sensitization to DNA-damaging agents.

Therefore, in this study, we focused on the effects of CDK inhibitors against solid tumors, such as cervical cancer, that are prevalent in some developing countries (20). The most common cause of refractoriness in cervical cancer treatment is resistance to radiation, whereas the treatment outcomes of ovarian cancer are dependent on the success of cytoreductive surgery and chemotherapy. If a patient has a tumor refractory to radiation, salvage chemotherapy can only elicit a response in a few cases. Currently, the majority of ongoing clinical trials for cervical cancer treatments investigate agents that target refractory disease. Hence, we selected CDK inhibitors as candidate target agents for refractory cervical cancer. In this study, we compared the anticancer effects of several CDK inhibitors currently undergoing clinical trials (data not shown). Among the inhibitors available, the two most potent candidates, AT7519 and SNS-032, were selected and further characterized in terms of cytotoxicity, senescence, and metastasis using cervical cancer cell lines and a human xenograft tumor model.

\section{Materials and methods}

Cells and reagents. The human cervical carcinoma cell lines, HeLa and ME-180, were obtained from the Korean Cell Line Bank (Seoul, Korea). The HeLa and ME-180 cells were maintained in Dulbecco's modified Easgle's medium (DMEM) and Roswell Park Memorial Institute (RPMI)-1640 medium (Welgene Inc., Korea), supplemented with $10 \%$ fetal bovine serum (FBS) (Capricorn Scientific GmbH, Germany) and 100 units of penicillin and streptomycin (Welgene). The cells were cultured in a humidified incubator containing $5 \% \mathrm{CO}_{2}$ at $37^{\circ} \mathrm{C}$. SNS-032 and AT7519 were purchased from Selleck Chemicals (Houston, TX, USA).

Cell viability assay. The HeLa and ME- 180 cells $\left(2-5 \times 10^{3}\right.$ cells/ well) were plated in 96-well plates and allowed to attach for $24 \mathrm{~h}$ prior to treatment. The cells were exposed to $0.001,0.01$,
$0.1,0.5,1$, or $10 \mu \mathrm{M}$ AT7519 and SNS-032 for $48 \mathrm{~h}$, and cell viability was measured using the resazurin reduction ratio (21). Resazurin solution was added to a final concentration of $50 \mu \mathrm{M}$, followed by incubation for $2-4 \mathrm{~h}$ and spectroscopy at A600 (Epoch, BioTek Instruments, Inc, Winooski, VT, USA). $\mathrm{IC}_{50}$ values were calculated using the ED50 Plus v1.0 online (http://www.sciencegateway.org/protocols/cellbio/drug/data/ ed50v10.xls).

Senescence-associated (SA) $\beta$-galactosidase assay. The HeLa cells $\left(1 \times 10^{4}\right)$ were plated in $35-\mathrm{mm}$ culture plates and treated with various concentrations of 0.05 or $0.1 \mu \mathrm{M}$ of AT7519 and SNS-032 for 3 days. The cells were fixed in $2 \%$ formaldehyde $/ 0.2 \%$ glutaraldehyde for $15 \mathrm{~min}$ at room temperature, and SA $\beta$-galactosidase staining was performed as previously described (22).

Irradiation. Delivery of $\gamma$-radiation was achieved using a dualsource ${ }^{137} \mathrm{Cs}$ unit at a dose rate of $3.2 \mathrm{~Gy} / \mathrm{min}$ with a GC-3000 Elan irradiator (MDS Nordion, Ottawa, Canada).

Western blot analysis. The cells were lysed in radioimmunoprecipitation assay (RIPA) buffer [50 $\mathrm{mM}$ Tris-Cl (pH 8.0), $150 \mathrm{mM} \mathrm{NaCl}, 0.1 \%$ SDS, $0.5 \%$ deoxycholic acid, $1 \%$ NP-40] containing a protease inhibitor and a phosphatase inhibitor cocktail and briefly sonicated. The protein content was measured using the Coomassie (Bradford) Protein assay kit (Thermo Fisher Scientific, Rockford, IL, USA). A total of $10-40 \mu \mathrm{g}$ of cell lysates were separated on 8,1012 , or $15 \%$ SDS-polyacrylamide gels and transferred to nitrocellulose membranes (Bio-Rad Laboratories, Inc., Hercules, CA, USA). The membranes were immunoblotted with antibodies against poly(ADP-ribose) polymerase (PARP)-1 (\#SC-8007, 1:2,000), p53 (\#SC-126, 1:2,000), Chk1 (\#SC-8408, 1:2,000), cyclin A (\#SC-239, 1:1,000), cyclin B1 (\#SC-245, 1:1,000), cyclin D1 (\#SC20044, 1:500), cyclin E (\#SC-247, 1:500), $\beta$-actin (\#SC-47778, 1:3,000) (all from Santa Cruz Biotechnology, Santa Cruz, CA, USA), caspase 3 (\#9662, 1:1,000), cleaved caspase 3 (\#4199, 1:1,000), phospho-p53 (ser15) (\#9284, 1:2,000), phospho-Chk1 (ser345) (\#2341, 1:1,000), phosphoChk2 (thr68) (\#2661, 1:2,000) (all from Cell Signaling Technology, Danvers, MA, USA), $\gamma$-H2AX (ser139) (\#05-636, 1:2,000), Chk2 (\#07-057, 1:2,000), phospho-ATM (ser1981) (\#05-740, 1:5,000) (Millipore, USA) and ATM (\#1549-1, 1:2,000) (Epitomics, Burlingame, CA, USA). HRP-conjugated secondary antibodies were obtained from Enzo Life Sciences (Farmingdale, NY, USA; ADI-SAB-100-J, ADI-SAB-300-J, 1:20,000). Chemiluminescence was detected using enhanced chemiluminescence detection reagents (Western Bright ${ }^{\mathrm{TM}}$ ECL kit, Advansta CO, USA).

BrdU assay. The HeLa and ME- 180 cells $\left(1 \times 10^{6}\right.$ cells) were plated in $100-\mathrm{mm}$ plates and exposed $1 \mu \mathrm{M}$ of CDK inhibitors for $16 \mathrm{~h}$. For combination treatments with $\gamma$-irradiation, $1 \mu \mathrm{M}$ AT7519 and $1 \mu \mathrm{M} \mathrm{SNS}-032$ were added to the cells $1 \mathrm{~h}$ prior to irradiation, and the cells were further incubated for $4 \mathrm{~h}$ following irradiation. BrdU was pulsed for $30 \mathrm{~min}$ before cells were harvested and fixed in $70 \%$ ethanol at $-20^{\circ} \mathrm{C}$ for $16 \mathrm{~h}$. The fixed cells were rinsed with phosphate-buffered saline (PBS) 3 times and incubated in $1.5 \mathrm{M} \mathrm{HCl}$ for $30 \mathrm{~min}$ 
at room temperature. After rinsing with PBS, the cells were resuspended in $100 \mu \mathrm{l}$ of $0.5 \%$ bovine serum albumin (BSA) in PBS and incubated for $10 \mathrm{~min}$. Fluorescein isothiocyanate (FITC)-conjugated BrdU antibody (\#11-5071-41, eBioscience/Thermo Fisher Scientific, Waltham, MA, USA) was added to cells, followed by $1 \mathrm{~h}$ of incubation prior to the analysis of the BrdU-positive cell profile using a flow cytometer [BD FACSCalibur (SN. E97501075), BD Biosciences, San Jose, CA, USA].

Migration and invasion assays. Eight-micrometer pore size Transwell filters (Corning Inc., Corning, NY, USA) were placed into 24-well plates and the upper chambers were covered with Matrigel for the invasion assay (BD Biosciences). Following treatment with $0.5 \mu \mathrm{M}$ AT7519 or $0.2 \mu \mathrm{M}$ SNS-032 for $24 \mathrm{~h}$,

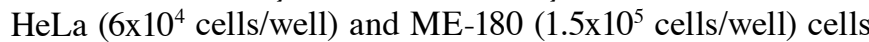
were suspended in $150 \mu \mathrm{l} \mathrm{FBS}$-free medium and seeded onto the filters. The lower chambers were filled with $500 \mu \mathrm{l}$ medium containing 10 or $20 \%$ FBS. At $16-48 \mathrm{~h}$ after seeding, the cells were fixed with ice-cold methanol for $5 \mathrm{~min}$, and stained with $0.5 \%$ crystal violet (Sigma-Aldrich, St. Louis, MO, USA) in $20 \%$ methanol for $10 \mathrm{~min}$ at room temperature. After washing with distilled water, the cells on the top side of the filter were removed with a cotton swab, then the number of migrated cells to the lower side were counted.

Tube formation assay. A total of 50,000 human umbilical vein endothelial cells (HUVECs; \#C-12203, Promo Cell, Heidelberg, Germany) were seeded into each well of a 24-well plate pre-coated with Matrigel; and added CDK inhibitors simultaneously. The cells were incubated overnight to allow the formation of tube-like structures. Endothelial cell tube formation was assessed using an IN Cell Analyzer imaging device (GE Healthcare Life Sciences, Pittsburgh, PA, USA). Tubular structures were quantified by counting the number of branches in each field $11 \mathrm{~h}$ after seeding.

Human xenograft tumor model. A total of 96 female BALB/c nude mice (5 weeks old, weighing $15 \mathrm{~g}$ ) were purchased from Orient Bio Co. (Seongnam, Korea) and allowed to acclimate to the new environment for 1 week before use. The room temperature and relative humidity were maintained at $22 \pm 3^{\circ} \mathrm{C}$ and $50 \pm 20 \%$, respectively. The mice were allowed access to water and food (Purina) ad libitum. For tumor generation, exponentially growing $1 \times 10^{6} \mathrm{ME}-180$ cells were injected subcutaneously into the right hind leg of each male BALB/c nude mouse. Tumor diameters were measured using a caliper, and tumor volumes were calculated with the following formula: $\mathrm{V}=0.523 \times \mathrm{AxB}^{2}$, where ' $\mathrm{A}$ ' is the longest diameter and ' $\mathrm{B}$ ' is the shortest diameter of the tumors. All animal experiments were conducted following a protocol approved by the Korea Institute of Radiological and Medical Sciences (KIRAMS) Animal Care and Use Committee (Reference no. KIRAMS 201400400). The weight of the mice upon sacrifice was $25 \mathrm{~g}$.

Tumor growth delay. The tumor-bearing mice were randomly divided into 6 groups of 8 mice per group and treated as follows: i) Control; ii) irradiation; iii) AT7519; iv) irradiation and AT7519; v) SNS-032; and vi) irradiation and SNS-032.
When the tumors were $6-7 \mathrm{~mm}$ in mean diameter, the mice were treated with AT7519 $(15 \mathrm{mg} / \mathrm{kg}$ once a day for 5 days for a 2-week duration) or SNS-032 $(15 \mathrm{mg} / \mathrm{kg}$ injected intraperitoneally every 2 days for a 2 -week duration). For the administration of radiation, the mice were lightly anesthetized with $5 \mathrm{mg} / \mathrm{kg}$ tiletamine/zolazepam (Virbac ZoletilTM 50; Virbac Lab., Carros, France), and the tumor-bearing legs were irradiated with a ${ }^{60} \mathrm{Co}$ irradiator (Thermatron 780 , Atomic Energy of Canada) at a dose rate of $1.3 \mathrm{~Gy} / \mathrm{min}$.

Assay of lung metastasis. The anti-metastatic potential in the 3 experimental groups: [i) control; ii) AT7519; and iii) SNS-032 (16 mice per group)] was tested using the spontaneous lung metastasis model. Briefly, $1 \times 10^{6} \mathrm{ME}-180$ cells per mouse were administered to the right thighs of 5-week-old male BALB/c nude mice. When the tumors reached a diameter of $6-7 \mathrm{~mm}$, the mice were randomly assigned to one of the three groups. Mouse lungs were removed on days 45 and 60 following treatment and fixed with Bouin's solution to count lung nodules under a polarizing light microscope with a $4 \mathrm{X}$ objective lens.

Statistical analyses. Data were analyzed using the Kruskal-Wallis non-parametric statistical test followed by the Mann-Whitney $\mathrm{U}$ test using Bonferroni correction to adjust the probability. Statistical analyses were performed using IBM SPSS Statistics version 20.

\section{Results}

AT7519 and SNS-032 inhibit cervical cancer cell growth via the induction of apoptosis, senescence, and cytostasis. To determine whether the CDK inhibitors, AT7519 and SNS-032, exert anticancer effects, cell viability assays were performed in two cervical cancer cell lines, HeLa and ME-180. As shown in Fig. 1A, cell growth was markedly inhibited in a dose-dependent manner. AT7519 inhibited HeLa and ME-180 cell growth with $\mathrm{IC}_{50}$ values of 0.352 and $0.599 \mu \mathrm{M}$, respectively, whereas SNS-032 inhibited HeLa and ME-180 cell growth with $\mathrm{IC}_{50}$ values of 0.183 and $0.206 \mu \mathrm{M}$, respectively. To determine the anticancer mechanisms of action of AT7519 and SNS-032, we first analyzed the cell cycle following treatment with either AT7519 or SNS-032. As shown in Fig. 1B, these CDK inhibitors dysregulated cell cycle progression and increased the sub-G1 cell population, particularly in the HeLa cells. Thus, we next examined whether these drugs induced the apoptosis of cervical cancer cells. As shown Fig. 1C, the cleavage of PARP-1 and caspase 3, classical markers of apoptosis, was increased following treatment with either AT7519 or SNS-032, in a dose-dependent manner. The HeLa cells were more susceptible to apoptosis by these CDK inhibitors. As CDK inhibition disrupts cell cycle progression, we investigated whether prolonged exposure to AT7519 or SNS-032 induces premature cellular senescence. The results of an SA $\beta$-galactosidase assay revealed that the numbers of senescent cells increased 2.5- and 3.5-fold following treatment with 0.05 or $0.1 \mu \mathrm{M}$ AT7519, respectively, for 3 days. Similar to AT7519, the number of SA $\beta$-galactosidase-stained cells increased by 2.4- and 3.7-fold following treatment with 0.05 or $0.1 \mu \mathrm{M}$ SNS-032, respectively (Fig. 1D). SA $\beta$-gal 
A HeLa

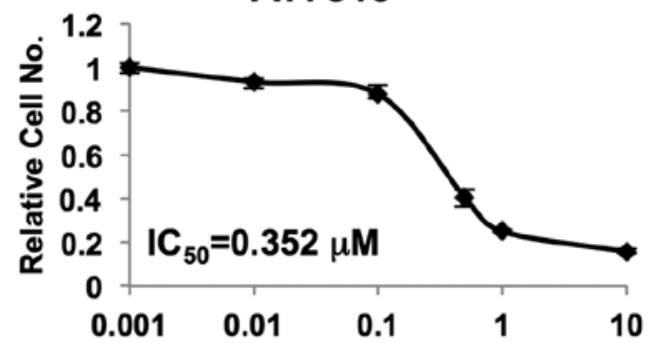

ME-180

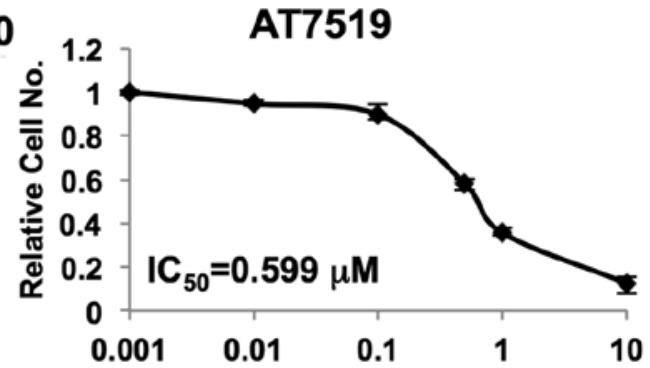

B

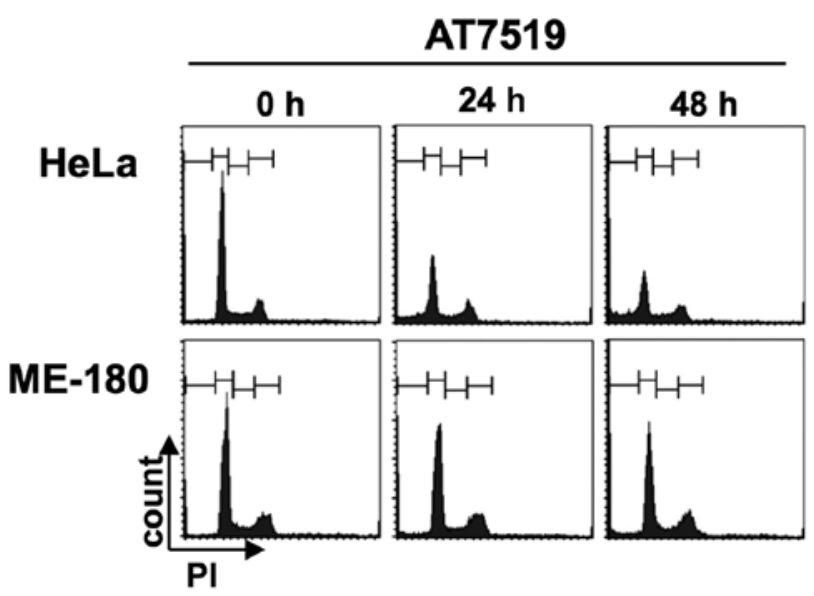

C

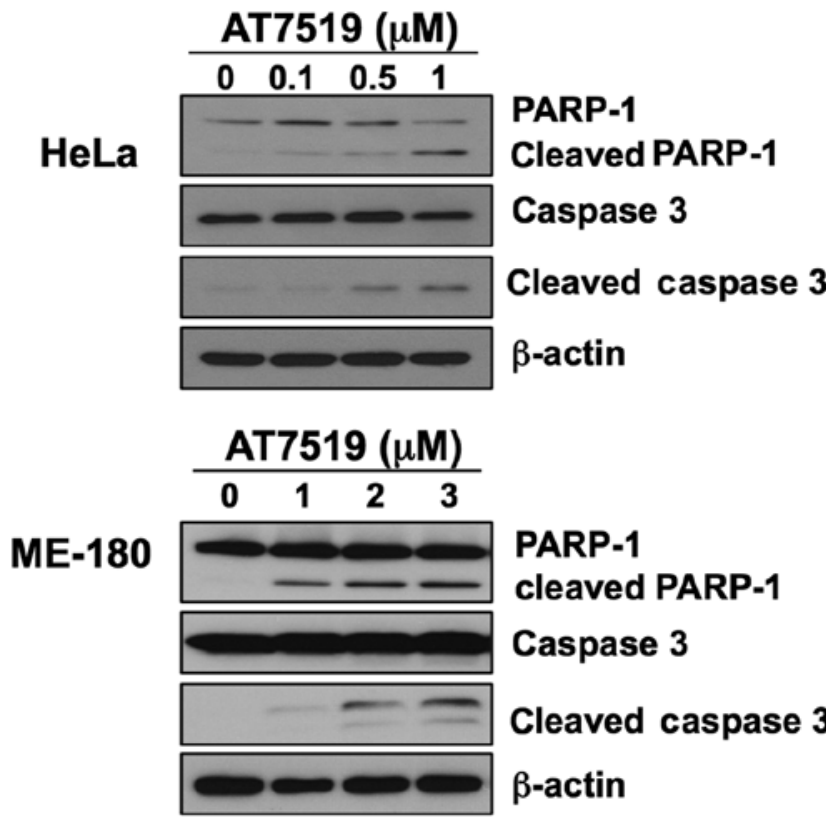

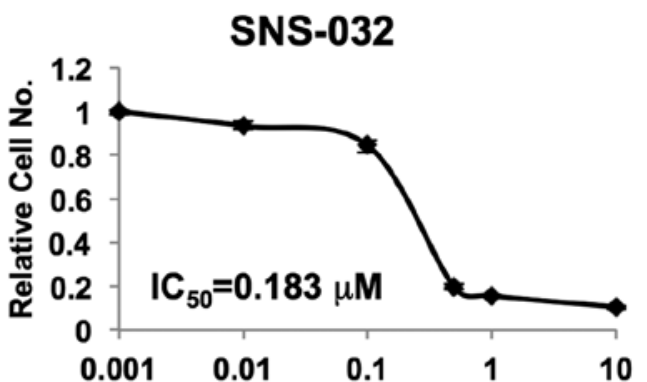

SNS-032

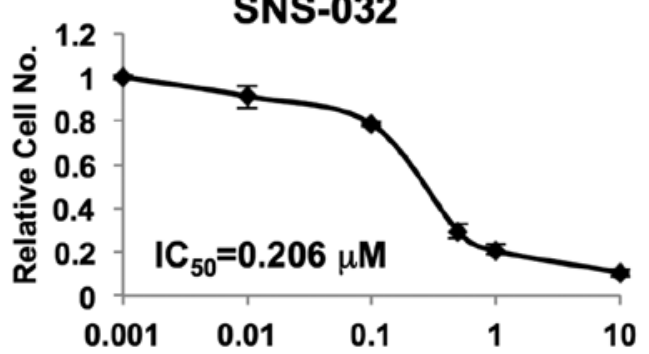

SNS-032

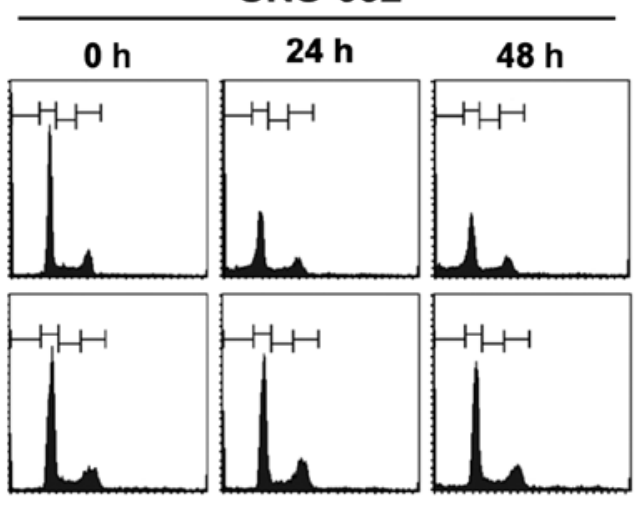

SNS-032 $(\mu \mathrm{M})$

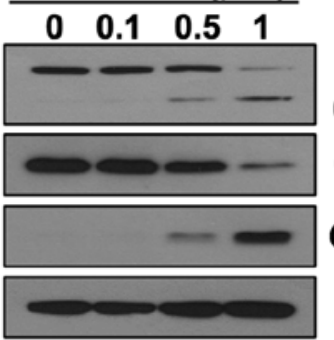

PARP-1

Cleaved PARP-1

Caspase 3

Cleaved caspase 3

$\beta$-actin
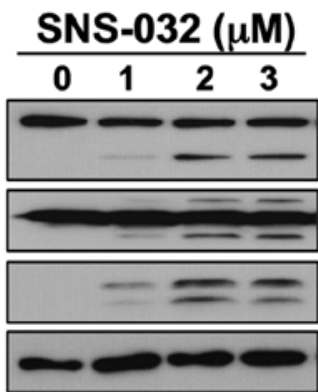

PARP-1

Cleaved PARP-1

Caspase 3

Cleaved caspase 3

$\beta$-actin

Figure 1. Anticancer effects of AT7519 and SNS-032. (A) Viability of HeLa and ME-180 cervical cancer cells following treatment with various concentrations of AT7519 or SNS-032 for 48 h. (B) Cell cycle analyses following treatment with $1 \mu \mathrm{M}$ AT7519 or $1 \mu \mathrm{M}$ SNS-032. (C) Western blot analyses following treatment with the indicated doses of cyclin-dependent kinase (CDK) inhibitors for apoptosis markers, poly(ADP-ribose) polymerase 1 (PARP-1), caspase 3, cleaved form of caspase 3 and $\beta$-actin. 
D

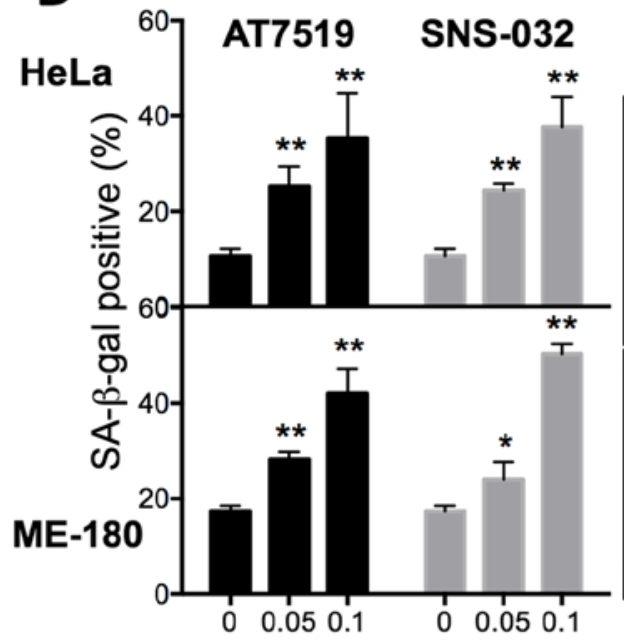

AT7519

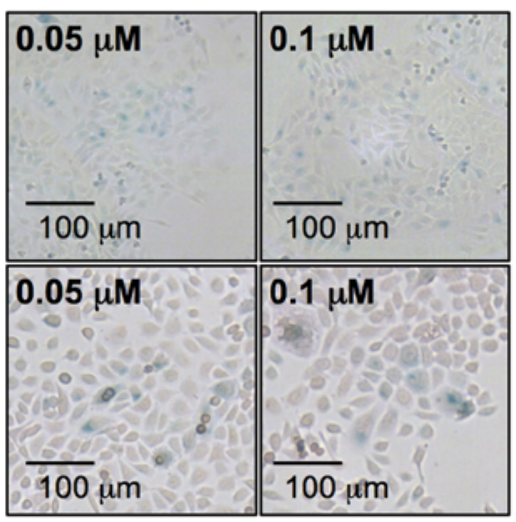

SNS-032

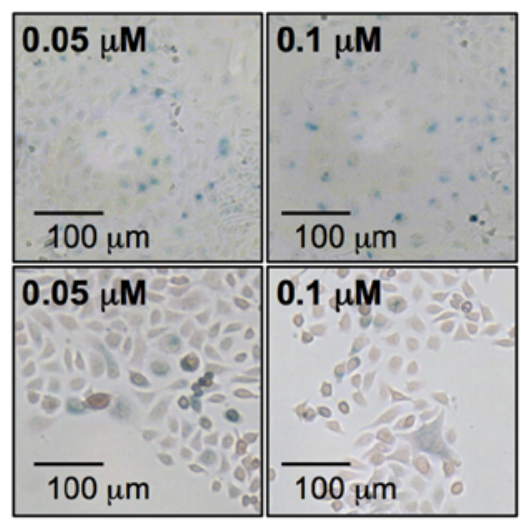

E

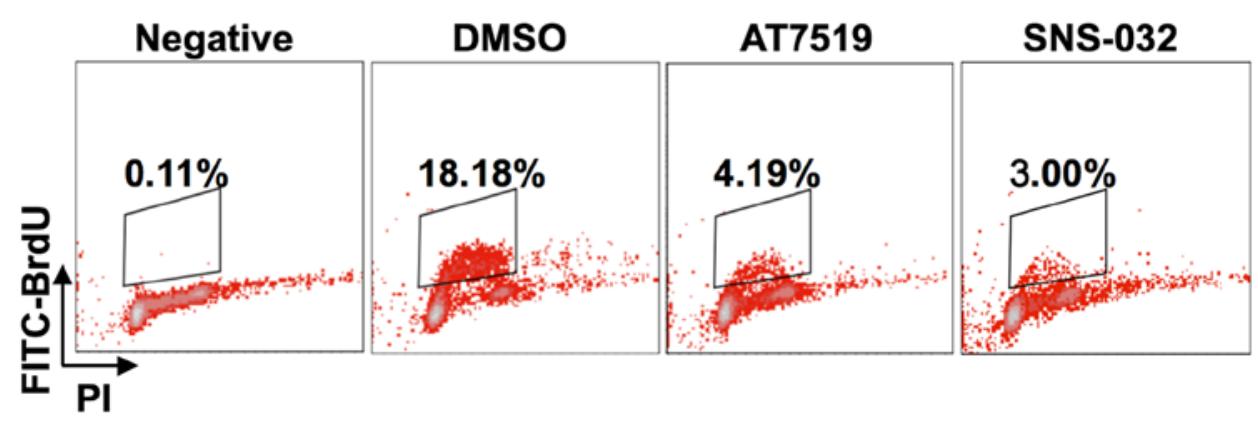

F

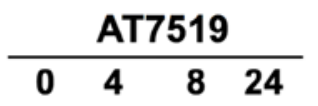

HeLa
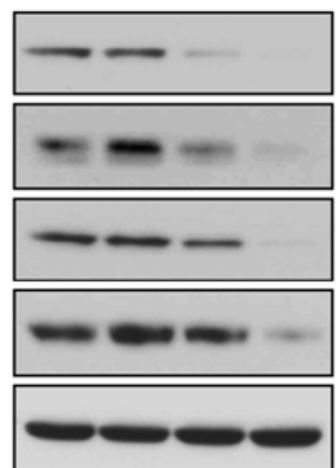

Cyclin A

Cyclin B1

ME-180
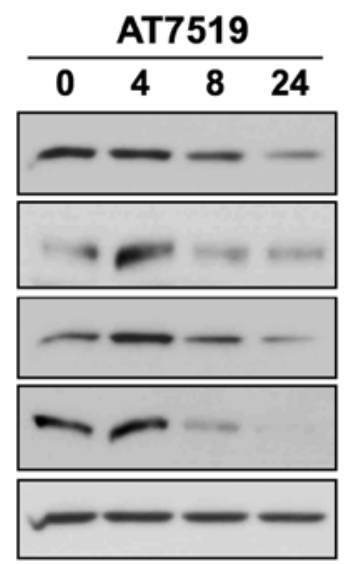

$\beta$-actin

(h)

Cyclin D1

Cyclin E

(h)

Cyclin D1

Cyclin E

Cyclin A

$\beta$-actin
Cyclin B1

Figure 1. Continued. (D) Senescence-associated $\beta$-galactosidase staining following treatment with the indicated concentrations of CDK inhibitors. (E) BrdU incorporation assay of ME-180 cells following treatment with $1 \mu \mathrm{M}$ AT7519 or $1 \mu \mathrm{M} \mathrm{SNS}-032$. (F) Western blot analyses following treatment with $1 \mu \mathrm{M}$ AT7519 or $1 \mu \mathrm{M}$ SNS-032 for cyclins. 


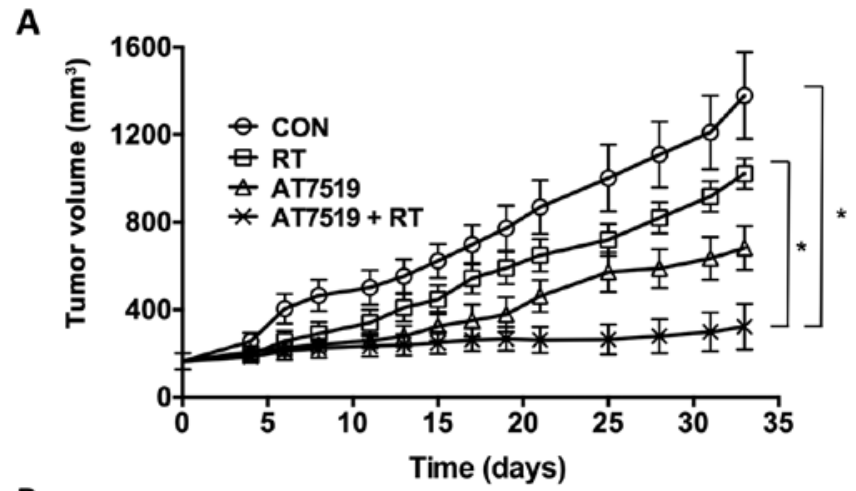

B

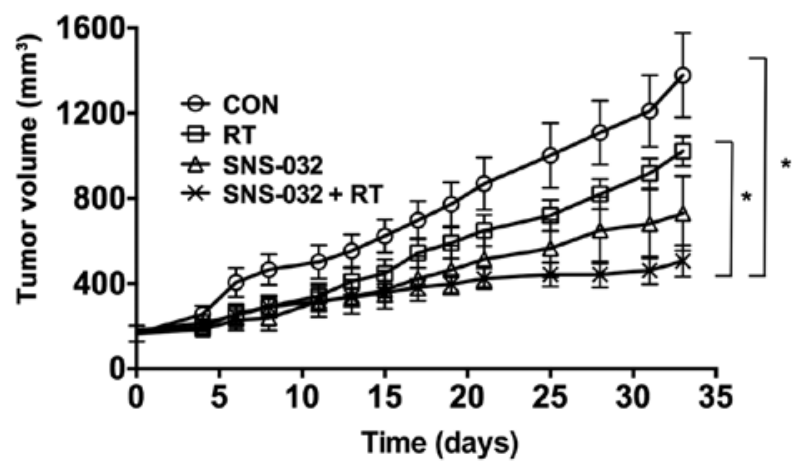

Figure 2. AT7519 and SNS-032 sensitize xenograft tumors to radiation in vivo. Tumor volume in mice with xenograft tumors was measured after treatment with a single dose of irradiation (9 Gy) in combination with (A) AT7519 or (B) SNS-032 as described in the Materials and methods. ${ }^{*} \mathrm{P}<0.05$.

positivity was also increased in the ME-180 cells following treatment with the CDK inhibitors. Finally, we measured the actively proliferating cell populations by BrdU incorporation assay. As shown in Fig. 1E, the population of BrdU-positive cells decreased from $18-4 \%$ following treatment with $1 \mu \mathrm{M}$ AT7519 and to $3 \%$ with $1 \mu \mathrm{M}$ SNS-032, respectively. The inhibition of cellular proliferation was dependent on the dose of the inhibitor applied (data not shown). As cyclins are the crucial cofactors of CDKs and seem to be deregulated in various cases of cervical cancer, we examined the levels of several key cyclins by western blot analysis. The levels of all the cyclins, (namely cyclin D1, E, A and B1) investigated were decreased by AT7519 and SNS-032 treatment (Fig. 1F). These results suggest that AT7519 and SNS-032 inhibited the growth of cervical cancer cells by inducing cell cycle deregulation, apoptosis, cellular senescence and cytostasis.

AT7519 and SNS-032 inhibit in vivo tumor growth and sensitize cervical cancer cells to radiation. To confirm the anticancer effects of these inhibitors in vivo and to examine their radiosensitizing effects, a human xenograft tumor was established. As shown in Fig. 2, the growth of subcutaneous ME-180 xenograft tumors in the legs of BALB/c nude mice were examined following exposure to various CDK inhibitors. The treatments commenced when the tumor volume reached $164-180 \mathrm{~mm}^{3}$. The volume of the control tumors (CON) progressively increased after reaching $400-500 \mathrm{~mm}^{3}$. The irradiation of the tumors of mice treated with a single exposure of 9 Gy (RT) suppressed tumor growth. The growth of the tumors of the mice treated with AT7519 or SNS-032 was slower than that of tumors of the controls or the tumors from the mice treated only with irradiation. The growth of the tumors of the mice that were both irradiated and treated with AT7519 or SNS-032 was significantly slower than that of the tumors of the mice treated with irradiation alone $(\mathrm{P}<0.05)$. Consequently, while the volume of the tumors of the untreated controls increased 2-fold in approximately 5 days, the volume of the tumors from the mice treated with irradiation doubled in 10 days. When irradiation was combined with treatment with AT7519 or SNS-032, the tumor-doubling time was delayed to 33 or 15 days, respectively.

AT7519 and SNS-032 modulate DNA damage response signaling and sensitize cells to radiation in vitro. As AT7519 and SNS-032 were shown to sensitize ME-180 xenograft tumors to radiation in vivo, we examined the mechanisms through which CDK inhibitors radiosensitize ME-180 cells. We first assessed the activation of the DNA damage signaling pathway. The ME-180 cells were treated with $1 \mu \mathrm{M}$ AT7519 or $1 \mu \mathrm{M}$ SNS-032 for $1 \mathrm{~h}$, followed by $\gamma$-irradiation. We found that the levels of $\gamma$-H2AX, a DNA double-strand break marker, were slightly increased in the CDK inhibitor-treated cells than in the cells treated with radiation alone (Fig. 3A). Although the phosphorylation levels of ATM and Chk2 were similar, p53 was markedly activated $4 \mathrm{~h}$ following both irradiation and treatment with AT7519 or SNS-032. In addition, AT7519 and SNS-032 completely abolished Chk1 phosphorylation induced by IR. These results suggest that AT7519 and SNS0-032 enhance cellular radiosensitivity via p53 activation and Chk1 inhibition. To determine whether AT7519 or SNS-032 accelerate apoptosis induced by radiation, the levels of apoptotic markers were examined by western blot analyses (Fig. 3B). The combination of AT7519 or SNS-032 with radiation increased the population of apoptotic cells. We then assessed the effects of the combination of radiation and CDK inhibitor treatment on S-phase cell cycle progression by BrdU incorporation assay (Fig. 3C). Although there was no inhibition of proliferation following treatment with radiation alone under our experimental conditions, the combination of AT7519 or SNS-032 and IR significantly decreased the population of cells in the S phase by $50 \%$.

AT7519 and SNS-032 inhibit cell migration and invasion. To evaluate the effects of AT7519 and SNS-032 on cancer metastasis, we performed cell migration and invasion assays. For the migration assay, the HeLa and ME-180 cells were seeded in Transwell ${ }^{\circledR}$ chambers and treated with $0.5 \mu \mathrm{M}$ AT7519 or $0.2 \mu \mathrm{M}$ SNS-032 for $24 \mathrm{~h}$. Both AT7519 and SNS-032 inhibited cervical cancer cell migration (Fig. 4A). The number of migratory HeLa cells was significantly reduced following treatment with AT7519 and SNS-032 to $0.38 \pm 0.08$ and $0.393 \pm 0.059$, respectively. The number of migratory ME-180 cells were also reduced following treatment with AT7519 $(0.220 \pm 0.045)$ and SNS-032 (0.111 \pm 0.010$)$. The inhibitory effects of AT7519 and SNS-032 on cell invasion were also confirmed (Fig. 4B). The number of invasive HeLa cells was reduced following treatment with $0.5 \mu \mathrm{M}$ AT7519 $(0.273 \pm 0.063)$ and $0.2 \mu \mathrm{M}$ SNS-032 $(0.334 \pm 0.045)$. The number of invasive ME-180 cells was also

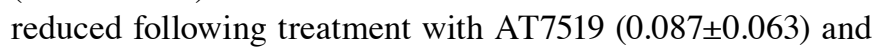
SNS-032 (0.070 \pm 0.004$)$. 

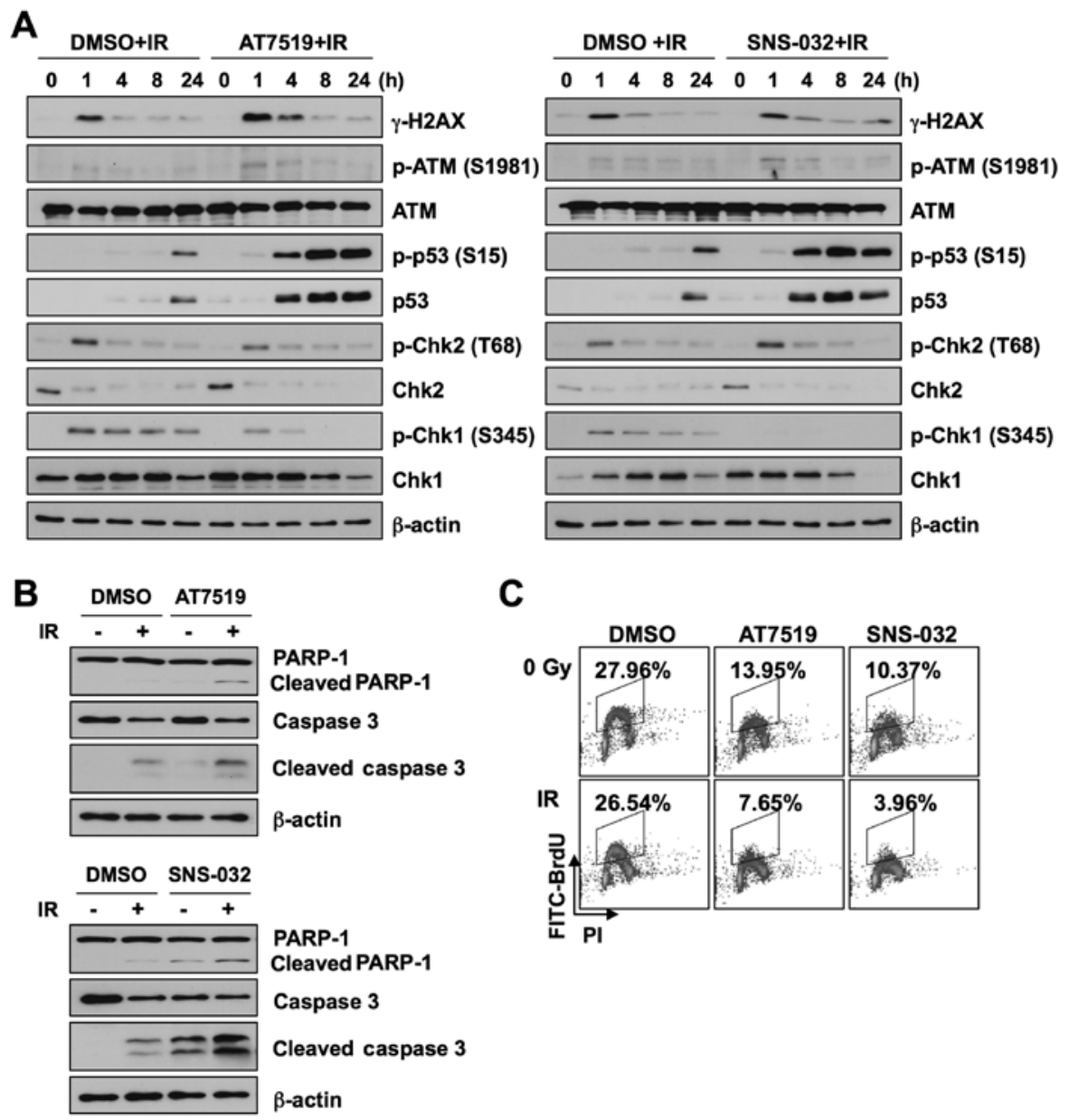

C

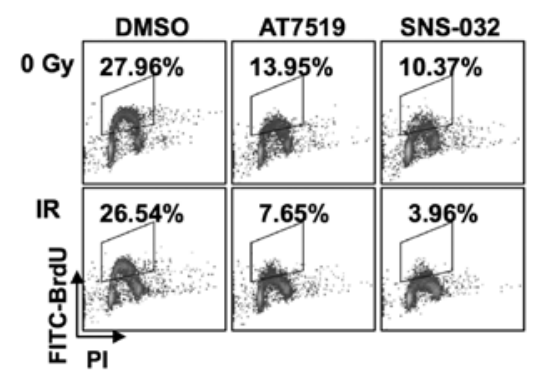

Figure 3. Cyclin-dependent kinase (CDK) inhibitors modulate DNA damage response signaling and sensitize cells to radiation in vitro. (A) Western blot analyses of ME-180 cells following treatment with 5 Gy of IR alone or in combination with $1 \mu \mathrm{M}$ AT7519 or $1 \mu \mathrm{M}$ SNS-032 for components of the DNA damage response signaling pathway. (B) Western blot analyses for apoptosis markers were performed $48 \mathrm{~h}$ after treatment as described in (A). (C) BrdU incorporation assay of ME-180 cells was performed $4 \mathrm{~h}$ following irradiation in combination with CDK inhibitors. IR, 5 Gy of ionizing radiation.
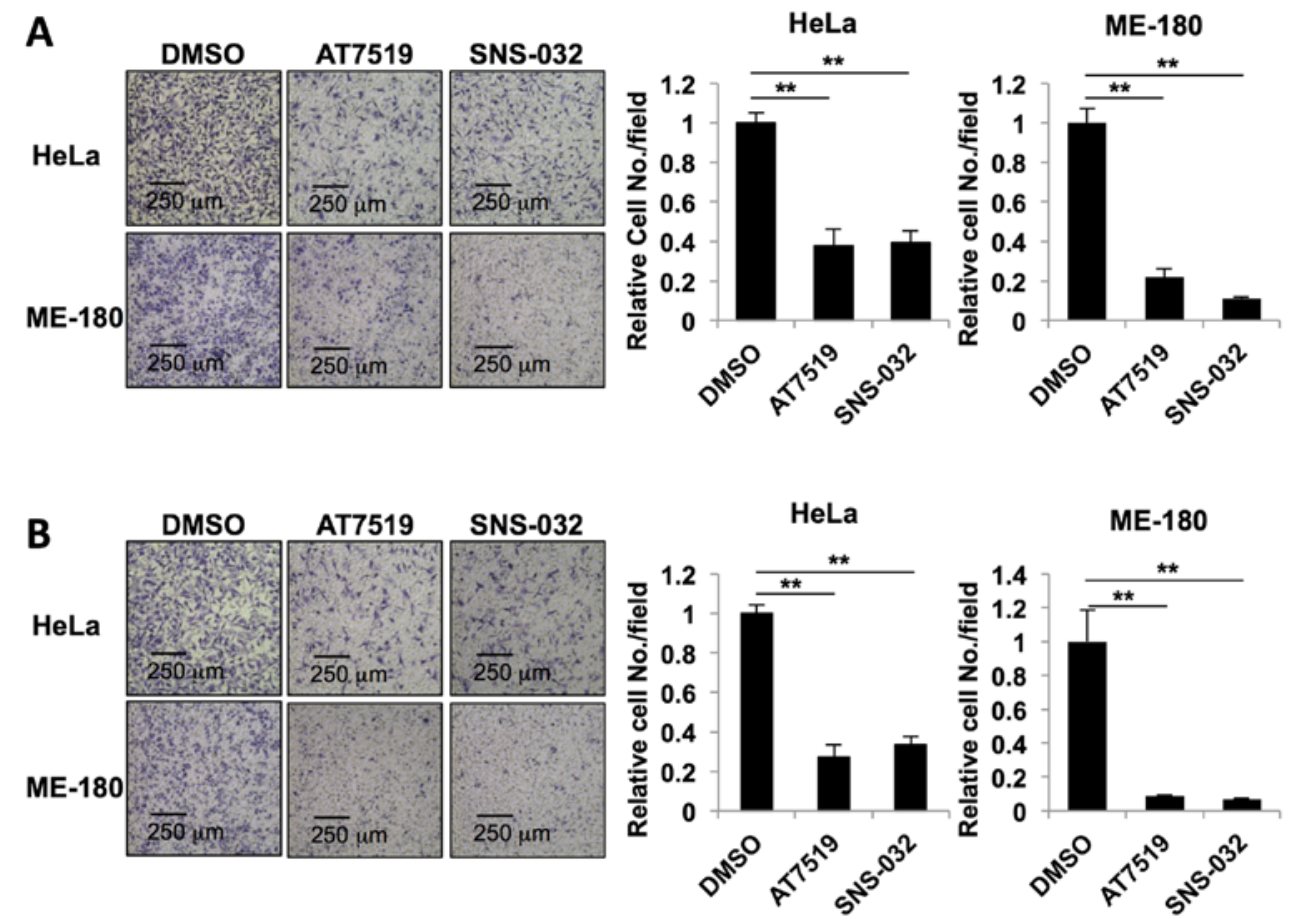

Figure 4. AT7519 and SNS-032 inhibit the migration and invasion of cervical cancer cells. (A) Migration assay and (B) invasion assay of cervical cancer cells were performed following treatment with AT7519 or SNS-032 as described in the Materials and methods. ${ }^{* *} \mathrm{P}<0.01$. 


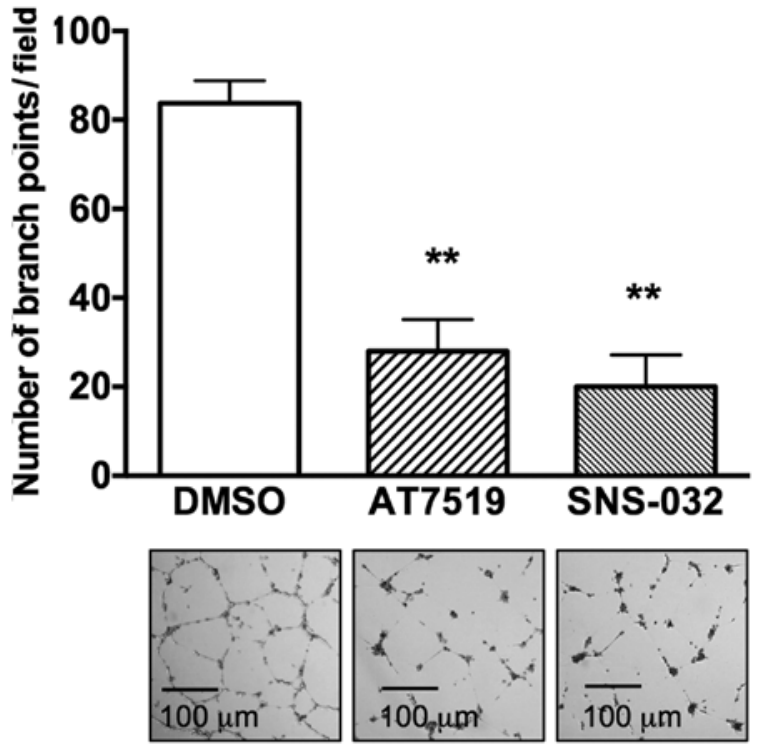

Figure 5. Anti-angiogenic effects of AT7519 and SNS-032. The numbers of branches in tube-like structures of human umbilical vein endothelial cells (HUVECs) was counted following treatment with $1 \mu \mathrm{M}$ AT7519 or $1 \mu \mathrm{M}$ SNS-032. Representative images are shown in the bottom panel. ${ }^{* *} \mathrm{P}<0.01$.

Anti-angiogenic effects of AT7519 and SNS-032. As angiogenesis accelerates tumor metastasis, the anti-angiogenic activities of the CDK inhibitors were measured. As shown in Fig. 5, HUVECs formed a well-organized tubular structure on Matrigel ${ }^{\circledR}$, representing functional activity of endothelial cells. However, both AT7519 and SNS-032 inhibited tube formation. Compared to $83.75 \pm 2.56$ branch points in the control group, the number of branch points per field decreased to $28 \pm 3.56$ $(\mathrm{P}<0.001)$ and $20 \pm 3.58(\mathrm{P}<0.001)$ in the presence of AT7519 and SNS-032, respectively. From this result, CDK inhibitors appeared to suppress tumor angiogenesis, in addition to suppressing the invasion and migration of cervical cancer cells.

Lung metastasis. To confirm the anti-metastatic activity of CDK inhibitors in vivo, a spontaneous lung metastasis model was employed using an ME-180 xenograft tumor model. Forty-five days after treatment, the average number of lung nodules of the control group was $12.83 \pm 1.89$. The number of lung nodules decreased to $7.75 \pm 0.94$ in the AT7519 group and to $9.12 \pm 0.79$ in the SNS-032 group. The anti-metastatic effects were more prominent after 60 days. Compared to $15.5 \pm 0.99$ in the control group, the number of lung nodules was $8.88 \pm 1.16$ $(\mathrm{P}<0.05)$ and $9.63 \pm 1.08(\mathrm{P}<0.05)$ in the AT7519 and SNS-032 groups, respectively (Fig. 6). These results confirm that both AT7519 and SNS-032 were able to suppress tumor metastasis to the lungs in vivo.

\section{Discussion}

In this study, we demonstrated that the CDK inhibitors, AT7519 and SNS-032, suppressed the growth of cancer cells in a dose-dependent manner (Fig. 1A). SNS-032 was more potent than AT7519, with a lower $\mathrm{IC}_{50}$ value. The mechanisms of growth inhibition can be summarized as cell cycle dysregulation, apoptosis, premature senescence and cytostasis. Although there was no a shift to a specific cell cycle phase, it seems likely that regulated cell cycle progression was inhibited by these CDK inhibitors (Fig. 1B). Quantitative measurements of the cleavage of PARP-1 and caspase 3, and SA $\beta$-galactosidase staining revealed that AT7519 and SNS-032 treatment induced the apoptosis and premature senescence of both HeLa and ME-180 cells (Fig. 1C and D). Although AT7519 and SNS-032 induced the apoptosis of HeLa cells, apoptosis did not seem to be a major mechanism of action in the ME-180 cells. However, there is a possibility that apoptosis could be induced in the ME-180 cells at higher concentration. It has been reported that CDK inhibitors exert cytostatic effects at lower concentrations, but induce apoptosis at higher concentrations. However, their mechanistic actions have not yet been clarified. Roscovitine has been reported to induce apoptosis at moderate cytotoxic concentrations by decreasing mitochondria membrane potential (23) or by reducing the amounts of the caspase inhibitor, XIAP (24). In addition, we investigated other possible anticancer mechanisms shown in Fig.1D-F rather than focusing on the apoptosis of HeLa cells. As CDKs are known to be master regulators of cell cycle progression, we hypothesized that cytostatic growth arrest may contribute to the anticancer effects of AT7519 and SNS-032, as reported for other anticancer agents (25). From the results of the BrdU incorporation

\section{Day 45}

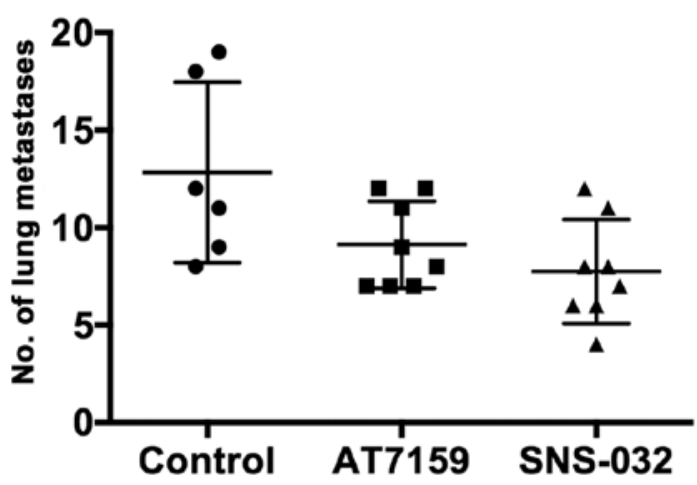

Day 60

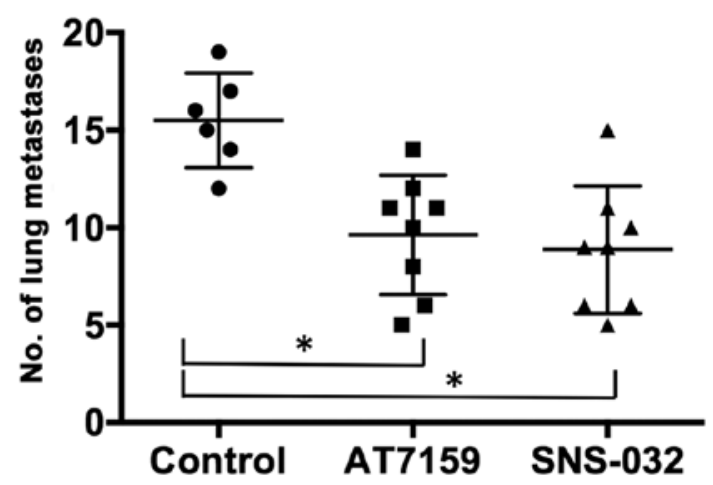

Figure 6. AT7519 and SNS-032 suppress lung metastasis in vivo. ME-180-cell xenograft tumors in mouse thighs generated spontaneous lung metastasis at 45 or 60 days after the grouping of the mice. Quantification of lung metastases in 6 groups was performed and analyzed statistically. ${ }^{*}<0.05$. 
assay, in the ME-180 cells treated with CDK inhibitors, the number of cells in the $\mathrm{S}$ phase decreased in a dose-dependent manner (Fig. 1E), suggesting that these CDK inhibitors exert cytostatic rather than cytotoxic effects on ME-180 cells. The depletion of cyclin D1, E, A and B1 (Fig. 1F) may be one of the crucial mechanisms of action of AT7519 and SNS-032, leading to cell cycle dysregulation and cytostasis. Growth arrest at various points of the cell cycle is known to eventually trigger cell death. Whether a drug is cytostatic or cytotoxic depends on the dose, the schedule of administration, the phase of the cell cycle during which the drug acts and in which the cell resides, and the cellular context (26). On the whole, it is suggested AT7519 and SNS-032 exert anticancer effects through cell cycle deregulation, premature senescence and cytostasis in both cervical cancer cells.

We then examined the synergistic effects of radiation and CDK inhibitors in vivo on tumor growth rate using a human xenograft tumor model (Fig. 2). Hence, we suggest that CDK inhibitors may be beneficial additions to standard chemoradiotherapy regimens for patients with cervical cancer. Initially, we hypothesized that the modulation of DNA double-strand break repair through the inhibition of HR and prolonged G(2)-M arrest may be a major sensitizing mechanism of AT7519 and SNS-032, as shown in other studies (27-29). However, although the $\gamma-\mathrm{H} 2 \mathrm{AX}$ levels were slightly elevated, DNA damage was efficiently repaired in the ME-180 cells following irradiation (Fig. 3A). By contrast, AT7519 or SNS-032 treatment induced p53 activation and inhibited the phosphorylation of Chk1 at Ser345 following irradiation (Fig. 3A). From these results, it can be concluded that the DNA damage-independent activation of p53 and cell cycle checkpoint deregulation occurs through the inhibition of Chk1 and contributes to radiaosensitization.

Metastasis is the most life-threatening event in patients with cancer. We assessed the effects of AT7519 and SNS-032 on the aggressiveness of cervical cancer cells in terms of invasion, angiogenesis and metastasis. The biological behavior of tumors is very important to consider during treatment, as although current modalities of cancer therapy have improved, they are insufficient to adequately treat aggressive tumors. The aggressiveness of tumors is usually defined by rapid invasion, accelerated angiogenesis and early metastasis. As shown in Fig. 4, CDK inhibitor treatment reduced the migration and invasion of HeLa and ME-180 cells. Anti-angiogenic therapy has been extensively utilized since bevacizumab was introduced as a treatment for patients with recurrent cervical cancer during the GOG 240 trial $(30,31)$. Both AT7519 and SNS-032 inhibited tube formation in HUVECs, which represent functional endothelial cells (Fig. 5). This finding suggests that $\mathrm{CDK}$ inhibitors may be possible candidates for use in a combination regimen of chemotherapeutics and irradiation for patients with recurrent cervical cancer.

Overall survival is often determined by the presence of distant organ metastases, such as of the liver, lungs and brain. Li et al suggested that lymph node-only metastases are better than organ metastases in patients with cervical cancer (32). Therefore, we assessed the effects of AT7519 and SNS-032 on organ metastases using a spontaneous metastasis model. The AT7519- and SNS-032-treated groups exhibited a statistically significant decrease in lung metastases from xenografted cervical cancer cells (Fig. 6). These findings are well in agreement with the results of the in vitro experiments (Figs. 4 and 5). Based on these findings, the CDK inhibitors, AT7519 and SNS-032, can enhance the efficacy of a combination of chemoand radiotherapies, thus impeding tumor cell progression for the treatment of advanced and metastatic cases of cervical cancer.

\section{Acknowledgments}

Not applicable.

\section{Funding}

This study was supported by a grant from the Korea Institute of Radiological and Medical Sciences (KIRAMS), funded by Ministry of Science and ICT (MSICT), Republic of Korea (50531-2018; 50458-2014).

\section{Availability of data and materials}

The datasets used and analyzed during the current study are available from the corresponding author on reasonable request.

\section{Authors' contributions}

MHK and JHJ conceived and designed the experiments; MAK, WK and HRJ performed the experiments; MAK, WK, HRJ, YJS, MK, and JJJ curated and analyzed the data; MAK, WK, HRJ, YJS, MHK, and JHJ wrote and edited manuscript. All authors have read and approved the nal version of the manuscript.

\section{Ethics approval and consent to participate}

All animal experiments were conducted following a protocol approved by the KIRAMS Animal Care and Use Committee (Reference no. KIRAMS 201400400).

\section{Patient consent for publication}

Not applicable.

\section{Competing interests}

The authors declare that they have no competing interests.

\section{References}

1. Malumbres M and Barbacid M: Mammalian cyclin-dependent kinases. Trends Biochem Sci 30: 630-641, 2005.

2. Bertoli C, Skotheim JM and de Bruin RA: Control of cell cycle transcription during G1 and S phases. Nat Rev Mol Cell Biol 14: 518-528, 2013.

3. Lim S and Kaldis P: Cdks, cyclins and CKIs: Roles beyond cell cycle regulation. Development 140: 3079-3093, 2013.

4. Swaffer MP, Jones AW, Flynn HR, Snijders AP and Nurse P CDK substrate phosphorylation and ordering the cell cycle. Cell 167: 1750-1761. e1716, 2016.

5. Malumbres M and Barbacid M: To cycle or not to cycle: A critical decision in cancer. Nat Rev Cancer 1: 222-231, 2001.

6. Malumbres M and Barbacid M: Cell cycle, CDKs and cancer: A changing paradigm. Nat Rev Cancer 9: 153-166, 2009.

7. Asghar U, Witkiewicz AK, Turner NC and Knudsen ES: The history and future of targeting cyclin-dependent kinases in cancer therapy. Nat Rev Drug Discov 14: 130-146, 2015. 
8. Senderowicz AM: Flavopiridol: The first cyclin-dependent kinase inhibitor in human clinical trials. Invest New Drugs 17: 313-320, 1999.

9. Santo L, Vallet S, Hideshima T, Cirstea D, Ikeda H, Pozzi S, Patel K, Okawa Y, Gorgun G, Perrone G, et al: AT7519, A novel small molecule multi-cyclin-dependent kinase inhibitor, induces apoptosis in multiple myeloma via GSK-3beta activation and RNA polymerase II inhibition. Oncogene 29: 2325-2336, 2010.

10. Chen EX, Hotte S, Hirte H, Siu LL, Lyons J, Squires M, Lovell S, Turner S, McIntosh L and Seymour L: A Phase I study of cyclindependent kinase inhibitor, AT7519, in patients with advanced cancer: NCIC Clinical Trials Group IND 177. Br J Cancer 111: 2262-2267, 2014

11. Chen R, Wierda WG, Chubb S, Hawtin RE, Fox JA, Keating MJ, Gandhi V and Plunkett W: Mechanism of action of SNS-032, a novel cyclin-dependent kinase inhibitor, in chronic lymphocytic leukemia. Blood 113: 4637-4645, 2009.

12. Heath EI, Bible K, Martell RE, Adelman DC and Lorusso PM: A phase 1 study of SNS-032 (formerly BMS-387032), a potent inhibitor of cyclin-dependent kinases 2, 7 and 9 administered as a single oral dose and weekly infusion in patients with metastatic refractory solid tumors. Invest New Drugs 26: 59-65, 2008.

13. Tong WG, Chen R, Plunkett W, Siegel D, Sinha R, Harvey RD, Badros AZ, Popplewell L, Coutre S, Fox JA, et al: Phase I and pharmacologic study of SNS-032, a potent and selective Cdk2, 7, and 9 inhibitor, in patients with advanced chronic lymphocytic leukemia and multiple myeloma. J Clin Oncol 28: 3015-3022, 2010.

14. Trovesi C, Manfrini N, Falcettoni M and Longhese MP: Regulation of the DNA damage response by cyclin-dependent kinases. J Mol Biol 425: 4756-4766, 2013.

15. Esashi F, Christ N, Gannon J, Liu Y, Hunt T, Jasin M and West SC: CDK-dependent phosphorylation of BRCA2 as a regulatory mechanism for recombinational repair. Nature 434 598-604, 2005

16. Finn K, Lowndes NF and Grenon M: Eukaryotic DNA damage checkpoint activation in response to double-strand breaks. Cell Mol Life Sci 69: 1447-1473, 2012.

17. Johnson N, Cai D, Kennedy RD, Pathania S, Arora M, Li YC, D'Andrea AD, Parvin JD and Shapiro GI: Cdk1 participates in BRCA1-dependent $S$ phase checkpoint control in response to DNA damage. Mol Cell 35: 327-339, 2009.

18. Laoukili J, Alvarez M, Meijer LA, Stahl M, Mohammed S, Kleij L, Heck AJ and Medema RH: Activation of FoxM1 during $\mathrm{G} 2$ requires cyclin A/Cdk-dependent relief of autorepression by the FoxM1 N-terminal domain. Mol Cell Biol 28: 3076-3087, 2008.

19. Alvarez-Fernández M, Halim VA, Krenning L, Aprelia M, Mohammed S, Heck AJ and Medema RH: Recovery from a DNA-damage-induced G2 arrest requires Cdk-dependent activation of FoxM1. EMBO Rep 11: 452-458, 2010.
20. Ferlay J, Soerjomataram I, Dikshit R, Eser S, Mathers C, Rebelo M, Parkin DM, Forman D and Bray F: Cancer incidence and mortality worldwide: Sources, methods and major patterns in GLOBOCAN 2012. Int J Cancer 136: E359-E386, 2015.

21. O'Brien J, Wilson I, Orton T and Pognan F: Investigation of the Alamar Blue (resazurin) fluorescent dye for the assessment of mammalian cell cytotoxicity. Eur J Biochem 267: 5421-5426, 2000.

22. Dimri GP, Lee X, Basile G, Acosta M, Scott G, Roskelley C, Medrano EE, Linskens M, Rubelj I and Pereira-Smith O: A biomarker that identifies senescent human cells in culture and in aging skin in vivo. Proc Natl Acad Sci USA 92: 9363-9367, 1995.

23. Arisan ED, Obakan P, Coker-Gurkan A, Calcabrini A, Agostinelli E and Unsal NP: CDK inhibitors induce mitochondria-mediated apoptosis through the activation of polyamine catabolic pathway in LNCaP, DU145 and PC3 prostate cancer cells. Curr Pharm Des 20: 180-188, 2014.

24. Mohapatra S, Chu B, Zhao X, Djeu J, Cheng JQ and Pledger WJ: Apoptosis of metastatic prostate cancer cells by a combination of cyclin-dependent kinase and AKT inhibitors. Int J Biochem Cell Biol 41: 595-602, 2009.

25. Sparreboom A, de Jonge MJ and Verweij J: The use of oral cytotoxic and cytostatic drugs in cancer treatment. Eur J Cancer 38: $18-22,2002$.

26. Rixe $\mathrm{O}$ and Fojo $\mathrm{T}$ : Is cell death a critical end point for anticancer therapies or is cytostasis sufficient? Clin Cancer Res 13: 72807287, 2007.

27. Kodym E, Kodym R, Reis AE, Habib AA, Story MD and Saha D: The small-molecule CDK inhibitor, SNS-032, enhances cellular radiosensitivity in quiescent and hypoxic non-small cell lung cancer cells. Lung Cancer 66: 37-47, 2009.

28. Raghavan P, Tumati V, Yu L, Chan N, Tomimatsu N, Burma S, Bristow RG and Saha D: AZD5438, an inhibitor of Cdk1,2, and 9, enhances the radiosensitivity of non-small cell lung carcinoma cells. Int J Radiat Oncol Biol Phys 84: e507-e514, 2012.

29. Storch K and Cordes N: The impact of CDK9 on radiosensitivity, DNA damage repair and cell cycling of HNSCC cancer cells. Int J Oncol 48: 191-198, 2016.

30. Tewari KS, Sill MW, Long HJ III, Penson RT, Huang H, Ramondetta LM, Landrum LM, Oaknin A, Reid TJ, Leitao MM et al: Improved survival with bevacizumab in advanced cervical cancer. N Engl J Med 370: 734-743, 2014.

31. Monk BJ and Tewari KS: Evidence-based therapy for recurrent cervical cancer. J Clin Oncol 32: 2687-2690, 2014.

32. $\mathrm{Li} \mathrm{H}, \mathrm{Wu} \mathrm{X}$ and Cheng $\mathrm{X}$ : Advances in diagnosis and treatment of metastatic cervical cancer. J Gynecol Oncol 27: e43, 2016. 